\title{
Simplified Signal Detection for BLAST Architecture with ML and DFE Detectors
}

\author{
Myung-Sun Baek, Byung-Su Kang, So-Young Yeo, \\ Young-Hwan You, and Hyoung-Kyu Song \\ uT Communication Research Institute, Sejong University, Seoul, Korea \\ sabman@sju.ac.kr, saturnus@sju.ac.kr, yeossoh@sju.ac.kr, \\ yhyou@sejong.ac.kr, songhk@sejong.ac.kr
}

\begin{abstract}
In this paper, a simplified signal detection technique is proposed for V-BLAST systems. In this proposed scheme, since the performance of V-BLAST system depends on the first sub-stream detection capability, $V$ probable streams are detected according to the first detected sub-stream of DFE detector and most probable stream is selected by likelihood test. And to reduce the computational complexity of DFE detector, a simple DFE detection scheme is also proposed. It has been shown that the proposed technique can detect the transmitted data more accurately than conventional DFE decoding scheme, and has very lower complexity than ML detector.
\end{abstract}

\section{Introduction}

Recently, there has been increasing interest in providing high quality and efficient broadband services over wireless and mobile links. One powerful performance enhancing technique, which holds the promise of offering significant increase in the system information capacity, is multiple input multiple output (MIMO) antenna technique [1]-3]. Although current communication architectures such as the Vertical Bell Laboratories layered space-time (V-BLAST) have demonstrated very high spectral efficiencies, they have not reached the available capacities. In particular, there is a wide gap between the performance obtained by original detection algorithm of V-BLAST and optimum performance algorithms [4. In general V-BLAST detection scheme which is called decision feedback equalization (DFE) or successive interference cancelation (SIC), since the performance depends on initial layer detection capability [5], the accurate detection of first layer is very important to increase an overall system performance.

In this paper, for more accurate detection of initial layer, simplified ML detection technique with DFE detection is proposed. At the same time, to reduce the computational quantity of the DFE detector we present simple DFE scheme with Cholesky factorization.

This paper is organized as follows. After describing the system model, general detection and proposed DFE detection is provided. Next, the proposed simplified signal detection algorithm is addressed. Simulation results and conclusions are finally showed. 


\section{System Description}

We consider $M$ transmit antennas and $N \geq M$ receive antennas. The data is demultiplexed in $M$ data layers of equal length. These layers are mapped into certain modulation symbols. The layers are transmitted over the $M$ transmit antennas simultaneously.

Let $\mathbf{x}=\left[\begin{array}{llll}x_{1} & x_{2} & \cdots & x_{M}\end{array}\right]$ denote the $M \times 1$ vector of transmit symbols, then the corresponding $N \times 1$ receive signal vector is given by

$$
\mathbf{r}=\mathbf{H x}+\mathbf{n}
$$

where $\mathbf{n}$ is zero-mean Gaussian noise with variance $\sigma_{n}^{2}$ and $\mathbf{H}=\left[\begin{array}{llll}\mathbf{h}_{1} & \mathbf{h}_{2} & \cdots & \mathbf{h}_{N}\end{array}\right]^{T}$ is an i.i.d random complex vector of multipath channel with each element of $\mathbf{h}_{n}=\left[\begin{array}{llll}h_{n 1} & h_{n 2} & \cdots & h_{n M}\end{array}\right]$.

\section{ML and DFE Detector}

\subsection{General ML and V-BLAST Detector}

Under the assumption of channel state information (CSI) perfectly known by the receiver, ML detection and decoding correspond to choosing the codeword $\mathbf{x}$ which determines the symbol combination with the smallest distance metric as a decision value as follows

$$
\hat{\mathbf{x}}_{M L}=\underset{\hat{\mathbf{x}}}{\operatorname{argmin}}\|\mathbf{r}-\mathbf{H} \cdot \hat{\mathbf{x}}\| .
$$

Theoretically, ML detection would be the optimum way of recovering the transmitted data at the receiver. But as the computational effort is of order $L^{M}$ in $L$-QAM system, ML detection is not feasible for real time implementations [5]. Therefore, suboptimum detection schemes are generally used.

The representative and general detection scheme is ordered SIC (OSIC) detection scheme [2. In the OSIC detector, the received signal vector $\mathbf{r}$ is multiplied by filter matrix $\mathbf{G}$ which is the Moore-Penrose pseudo-inverse denoted by $(\cdot)^{\dagger}$ of the channel matrix [2]. The zero-forcing (ZF) Moore-Penrose pseudo-inverse matrix is

$$
\mathbf{G}_{Z F}=\mathbf{H}^{\dagger}=\left(\mathbf{H}^{H} \mathbf{H}\right)^{-1} \mathbf{H}^{H}
$$

And in the MMSE detector, minimizing the mean squared error (MSE) between the actually transmitted symbols and the output of a linear detector leads to the filter matrix

$$
\mathbf{G}_{M M S E}=\left(\mathbf{H}^{H} \mathbf{H}+\sigma_{n}^{2}\right)^{-1} \mathbf{H}^{H} .
$$

With the definition of a $(M+N) \times M$ extended channel matrix $\underline{\mathbf{H}}$ through

$$
\underline{\mathbf{H}}=\left[\begin{array}{l}
\mathbf{H} \\
\sigma_{n} \mathbf{I}_{M}
\end{array}\right],
$$


the MMSE filter given by Eqn. (4) can be rewritten as follows [5]

$$
\mathbf{G}_{M M S E}=\left(\underline{\mathbf{H}}^{H} \underline{\mathbf{H}}\right)^{-1} \underline{\mathbf{H}}^{H} \text {. }
$$

Assume that sub-stream $i$ yields the smallest estimation error or, equivalently, the largest signal-to-noise ratio (SNR) after linear nulling of the interference. It can be concluded that this sub-stream is associated with the row $\mathbf{g}^{(i)}$ of $\mathbf{G}$ that has minimum Euclidean norm, because this vector brings out the smallest noise enhancement. So, during the first step of the algorithm, only the decision static

$$
\hat{x}_{i}=\mathbf{g}^{(i)} \mathbf{r}=\mathbf{g}^{(i)}(\mathbf{H x}+\mathbf{n})=x_{i}+\eta_{i}
$$

with the effective noise $\eta_{i}=\mathbf{g}^{(i)} \mathbf{n}$ is used to find an estimate $\hat{x}_{i}$ for the transmit signal $x_{i}$.

The interference caused by this signal is then subtracted from the receive signal vector $\mathbf{r}$ and the $i$-th column is removed from the channel matrix, leading to a new system with only $M-1$ transmit antennas. This procedure consisting of nulling and cancelling is repeated for the reduced system until all signals are detected [2], [5]. In this procedure, the pseudo-inverse matrix calculation of the channel matrix is required in every layer detection. For example, the VBLAST system with 8 transmit antennas requires 8 times pseudo-inverse matrix calculations. Therefore, as the number of transmit antenna is increased, the decoding complexity is highly increased. To reduce this high complexity, the decomposition theory is applied in V-BLAST decoding.

\subsection{Proposed DFE Detector with Cholesky Factorization}

In this subsection, the sorted MMSE DFE detector with Cholesky factorization is proposed.

Let $\mathbf{R}=\mathbf{H}^{H} \mathbf{H}$ denote the cascade of $\mathbf{H}$; since $\mathbf{R}$ is Hermitan, it possesses a unique Cholesky factorization of the form $\mathbf{R}=\mathbf{D}^{H} \mathbf{D}$, where $\mathbf{D}$ is lower triangular with real diagonal elements. Multiplying the received signal $\mathbf{r}$ in Eqn. (1) by the unitary matrix $\mathbf{D}^{-H} \mathbf{H}^{H}$ yields 5 .

$$
\mathbf{z}=\mathbf{D}^{-H} \mathbf{H}^{H} \mathbf{r}=\mathbf{D} \mathbf{x}+\boldsymbol{\eta}
$$

where $\boldsymbol{\eta}=\mathbf{D}^{-H} \mathbf{H}^{H} \mathbf{n}$.

The whole proposed DFE algorithm based on Cholsky factoriztion is described as follows.

Step 1. The column of the channel matrix is sorted according to MMSE criterion in Eqn. (4). In this step, the MMSE criterion calculates $\left\|\mathbf{G}_{i}\right\|^{2}$ in Eqn (4) for $i=1,2, \cdots, M$, sorts them from the largest to the smallest and saves the sorted column index in sequence $\mathbf{k}, \mathbf{k}=\left\{k_{1}, k_{2}, \cdots, k_{M}\right\}$. Finally rearrange the columns of $\mathbf{H}$ according to the sorted index sequence $\mathbf{k}$

$$
\mathbf{H}_{\text {sort }}=\left[\mathbf{H}_{k_{1}} \mathbf{H}_{k_{2}} \cdots \mathbf{H}_{k_{M}}\right]
$$

where $\mathbf{H}_{k_{i}}$ is $\left[\begin{array}{llll}H_{1, k_{i}} & H_{2, k_{i}} & \cdots & H_{N, k_{i}}\end{array}\right]^{T}$. 
Step 2. The rearranged channel matrix $\mathbf{H}_{\text {sort }}$ in Eqn. (9) is extended to $\underline{\mathbf{H}}_{\text {sort }}$ and Cholesky factorization is executed as follows

$$
\underline{\mathbf{H}}_{\text {sort }}^{H} \underline{\mathbf{H}}_{\text {sort }}=\underline{\mathbf{R}}_{\text {sort }}=\underline{\mathbf{D}}^{H} \underline{\mathbf{D}} \text {. }
$$

Step 3. In extended $\underline{\mathbf{D}}$ of Eqn. (10), the required part $\underline{\mathbf{D}}^{\prime}$ which is $M$ by $M$ matrix is picked and signal detection is executed as follows

$$
\begin{aligned}
\mathbf{z}^{\prime} & =\left(\underline{\mathbf{D}}^{\prime}\right)^{-H} \mathbf{H}_{\text {sort }}^{H} \mathbf{r}=\underline{\mathbf{D}}^{\prime} \mathbf{x}+\boldsymbol{\eta}^{\prime} \\
& =\left[\begin{array}{cccc}
d_{1,1} & 0 & \cdots & 0 \\
d_{2,1} & d_{2,2} & \cdots & 0 \\
\vdots & \vdots & \ddots & \vdots \\
d_{M, 1} & d_{M, 2} & \cdots & d_{M, M}
\end{array}\right]\left[\begin{array}{l}
x_{k_{1}} \\
x_{k_{2}} \\
\vdots \\
x_{k_{M}}
\end{array}\right]+\left[\begin{array}{l}
\eta_{1} \\
\eta_{2} \\
\vdots \\
\eta_{M}
\end{array}\right]
\end{aligned}
$$

step 4 . The detected signal $\hat{\mathbf{x}}=\left(\hat{x}_{k_{1}} \hat{x}_{k_{2}} \cdots \hat{x}_{k_{M}}\right)$ is rearranged according to the order of transmit antenna by using index sequence $\mathbf{k}$.

In this process, the pseudo-inverse matrix calculation and sorting procedure of channel matrix are required only once.

\section{Simplified Signal Detection}

The proposed ML technique consists of three steps. In first step, $V$ sub-streams are detected, which are first outputs of DFE detector. Next, $V$ streams are detected according to the detected $V$ sub-streams in first step. In this step, the DFE decoding process is executed $V$ times. In final step, most probable stream among $V$ streams in second step is selected by likelihood test. The whole algorithm is described as follows.

Step 1. Detecting $V$ sub-streams at the first detection order of sorted DFE detector: The first detected sub-stream of Eqn. (11) can be presented as follows

$$
\tilde{x}_{k_{1}}=z_{1}^{\prime} / d_{1,1}=\left(d_{1,1} x_{k_{1}}+\eta_{1}\right) / d_{1,1}
$$

and $V$ probable sub-streams are determined. It can be expressed as follows

$$
\hat{\mathbf{x}}_{k_{1}}=Q^{(V)}\left(\tilde{x}_{k_{1}}\right)
$$

where $Q^{(V)}(k)$ is decision function which determines $V$ symbols of $L$-QAM or $L$-PSK system $(V \in L)$ by checking the Euclidean distance from $k$ to each symbol, and $\hat{\mathbf{x}}_{k_{1}}=\left\{\hat{x}_{k_{1}}^{(1)}, \ldots, \hat{x}_{k_{1}}^{(v)}, \ldots, \hat{x}_{k_{1}}^{(V)}\right\}$ where $\hat{x}_{k_{1}}^{(v)}$ is $v$-th estimated symbol whose Euclidean distance from $\tilde{x}_{k_{1}}$ is $v$-th. Fig. 1 1 shows the process of $Q^{(V)}\left(\tilde{\mathbf{x}}_{k_{1}}\right)$ when $V=4$. 


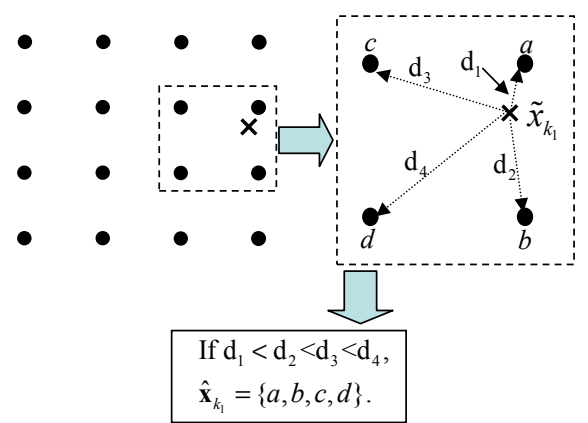

Fig. 1. Process of $Q^{(V)}\left(\tilde{\mathbf{x}}_{k_{1}}\right)$ when $V=4$

Step 2. Determining $V$ sub-stream by using sorted DFE detector according to detected $V$ sub-streams of step 1:

$$
\begin{aligned}
& \hat{x}_{k_{2}}^{(1)}=Q\left[\left(z_{2}-d_{2,1} \hat{x}_{k_{1}}^{(1)}\right) / d_{2,2}\right], \cdots \\
& \hat{x}_{k_{M}}^{(1)}=Q\left[\left(z_{M}-\sum_{i=1}^{M-1} d_{M, i} \hat{x}_{k_{i}}^{(1)}\right) / d_{M, M}\right], \\
& \vdots \\
& \hat{x}_{k_{2}}^{(v)}=Q\left[\left(z_{2}-d_{2,1} \hat{x}_{k_{1}}^{(v)}\right) / d_{2,2}\right], \cdots \\
& \hat{x}_{k_{M}}^{(v)}=Q\left[\left(z_{M}-\sum_{i=1}^{M-1} d_{M, i} \hat{x}_{k_{i}}^{(v)}\right) / d_{M, M}\right], \\
& \vdots \\
& \hat{x}_{k_{2}}^{(V)}=Q\left[\left(z_{2}-d_{2,1} \hat{x}_{k_{1}}^{(V)}\right) / d_{2,2}\right], \cdots \\
& \hat{x}_{k_{M}}^{(V)}=Q\left[\left(z_{M}-\sum_{i=1}^{M-1} d_{M, i} \hat{x}_{k_{i}}^{(V)}\right) / d_{M, M}\right] .
\end{aligned}
$$

In this step, the DFE decoding process is executed $V$ times.

Step 3. Selecting the most probable stream among $V$ streams in step 2: In this step, final stream maximizing likelihood is selected among $V$ streams from second step.

To minimize the probability of error for a particular stream, the receiver should select $\hat{\mathbf{x}}^{(v)}=\left[\hat{x}_{k_{1}}^{(v)}, \hat{x}_{k_{2}}^{(v)}, \ldots, \hat{x}_{k_{M}}^{(v)}\right]$ that maximizes the a posteriori logprobability (LP)

$$
\begin{aligned}
\operatorname{LP}\left(\hat{\mathbf{x}}^{(v)} \mid \mathbf{r}\right) & =\operatorname{logp}\left(\hat{\mathbf{x}}^{(v)} \mid \mathbf{r}\right) \\
& =\log p\left(\hat{\mathbf{x}}^{(v)}\right)+\log p\left(\mathbf{r} \mid \hat{\mathbf{x}}^{(v)}\right)-\log p(\mathbf{r}) \\
& =\operatorname{LP}\left(\hat{\mathbf{x}}^{(v)}\right)+\operatorname{LP}\left(\mathbf{r} \mid \hat{\mathbf{x}}^{(v)}\right)-\operatorname{LP}(\mathbf{r})
\end{aligned}
$$

where the first term corresponds to a prior information on $\hat{\mathbf{x}}^{(v)}$, the second term corresponds to the extrinsic information (or likelihood function), and the third term is just a normalization factor [7].

Since consideration of a prior information on $\hat{\mathbf{x}}^{(v)}$ is not required, the receiver needs to evaluate the second term, the likelihood function. The corresponding likelihood can be expressed as 


$$
L P\left(\mathbf{r} \mid \hat{\mathbf{x}}^{(v)}\right)=\left[\left(\mathbf{r} \cdot \mathbf{H} \cdot \hat{\mathbf{x}}^{(v)}\right)^{H}\left(\mathbf{r} \cdot \mathbf{H} \cdot \hat{\mathbf{x}}^{(v)}\right)\right] .
$$

Note that maximizing likelihood function is equivalent to minimize Euclidean distance between $\mathbf{r}$ and $\mathbf{H} \hat{\mathbf{x}}^{(v)}$. Thus, final decision value can be obtained as 8 ]

$$
\hat{\mathbf{x}}_{\text {final }}=\underset{\hat{\mathbf{x}}^{(v)}}{\operatorname{argmin}}\left\|\mathbf{r}-\mathbf{H} \cdot \hat{\mathbf{x}}^{(v)}\right\| .
$$

Since this simplified ML technique considers only $V$ candidate streams, much lower complexity is need than that of conventional ML detection scheme.

The introduced ML detection technique can use any other DFE detector such as OSIC and QR decomposition as well as the Proposed DFE detector.

\section{$5 \quad$ Simulation Results and Discussions}

To evaluate the performance of the proposed technique, V-BLAST system with QPSK modulation is considered. It is assumed that the receiver acquires the perfect CSI.

Fig. 2 shows the bit error rate (BER) performance of the system with $M=$ $N=4$. It can be seen that the proposed sorted ZF and MMSE DFE detectors with Cholesky factorization achieve the similar performance as the ZF and MMSE SIC schemes, with significantly lower complexity.

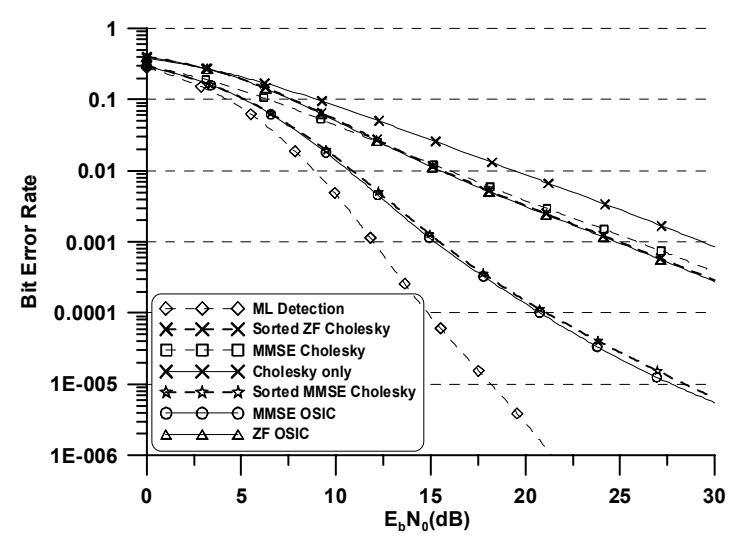

Fig. 2. BER performance comparison for $M=N=4$

Fig. 3] shows the BER performance of the proposed technique with $M=N=3$ according to the number of $V$. As expected, the more the number of $V$ is adopted, the better BER performance is acquired. In the case of $V=4$, the performance of proposed method can be dramatically improved due to reduction of error propagation. 


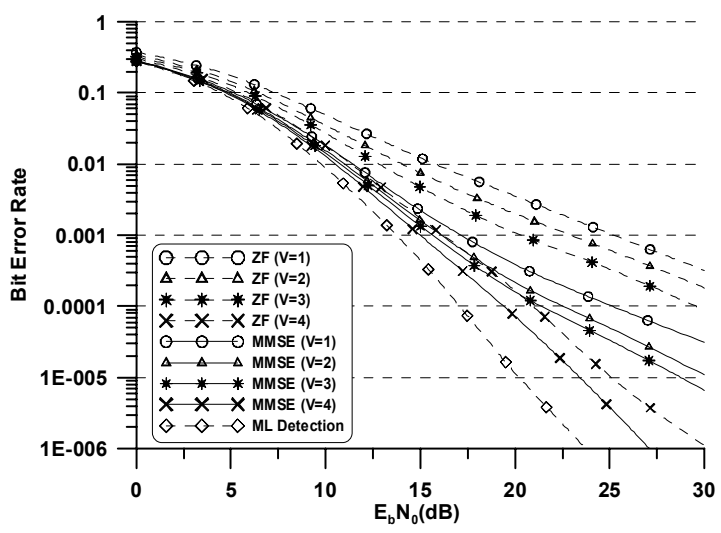

Fig. 3. BER performance of proposed technique with both ZF and MMSE detections according to the number of $V$

Fig. 4 illustrates the effect of the number of transmit antennas on the BER performance of proposed ML with $\mathrm{V}=4$ and conventional SIC. In this figure, it can be easily observed that the performance of the proposed technique is better than that of classical VBLAST detection and as the number of transmit antennas is increased, the performance differences are larger due to the efficient diminution of noise enhancement.

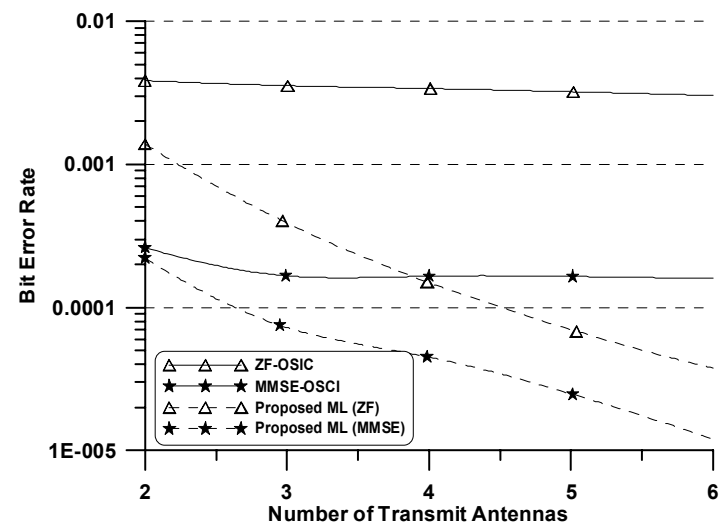

Fig. 4. BER performance according to the number of transmitting antennas for proposed ML $(V=4)$ and conventional OSIC

\section{Conclusions}

Since the performance of V-BLAST system is limited by the first detected substream due to error propagation, we propose the simplified ML detection technique which can detect the first sub-stream more accurately. And to reduce 
the complexity of sorted DFE detector, simplified DFE detector with Cholesky factorization is presented. The proposed DFE detector achieves the similar performance as the conventional SIC scheme. Simulation results show that the performance of the V-BLAST system can be improved by adopting the proposed ML detection technique, and the decoding complexity and system performance can be controlled by adjusting the number of $V$.

\section{Acknowledgements}

This work is financially supported by the Ministry of Education and Human Resources Development (MOE), the Ministry of Commerce, Industry and Energy (MOCIE) and the Ministry of Labor (MOLAB) through the fostering project of the Lab of Excellency and is supported by MIC Frontier R\&D Program in KOREA.

\section{References}

1. G. J. Foschini, "Layered space-time architecture for wireless communications in a fading environment when using multi-element antennas," Bell Labs Technical Journal, vol. 1, no. 2, pp. 41-59, Autumn 1996.

2. P. W. Wolniansky, G. J. Foschini, G. D. Golden, and R. A. Valenzuela, "V-BLAST: An architecture for achieving very high data rate over rich-scattering wireless channels," Proc. of ISSSE'98, pp.295-300, Italy, September 1998.

3. W. Y. Tao, R. S. Cheng, and K. B. Letaief, "Adaptive space-time coding system in fading channels," Proc. of VTC 2001 Spring, vol. 1, pp.103 - 107, May 2001.

4. A. Bhargave, R. Figueiredo, and T. Eltoft, "A detection algorithm for the V-BLAST system ," Proc. of GLOBECOM '01, vol. 1, pp.494 - 498, November 2001.

5. R. Bohnke, D. Wudden, K. Kammeyer, "Reduced complexity MMSE detection for BLKAST architectures," Proc. of GLOBECOM 2003, pp.2258-2262, September 2003.

6. A. Vielmon, Y. G. Li and J. R. Barry, "Performance of Alamouti transmit diversity over time-varying rayleigh-fading channels," IEEE Trans. Wireless Comm., vol. 3, pp. 1369-1373, September 2004.

7. J. Zhao, X. Xu, C. Wu, and K. Ng, "A novel joint ML and DFE scheme for V-BLAST system," Proc. WCNM 2005, vol. 1, pp.472-475, September 2005.

8. H. Sung, K. B. L and J. W. Kang "A simplified maximum likelihood detection scheme for MIMO systems," VTC 2003, vol. 1, pp. 419-423, Octorber 2003. 